\title{
Estudio de la corriente de transición en GMAW (MIG/MAG)
}

\author{
Study of the transition current in GMAW (MIG/MAG)
}

\author{
Andrés Mauricio Moreno-Uribe ${ }^{1^{*}} \quad$ Carlos Gregório Augusto Romanelli Sales ${ }^{1}$ \\ Pedro Henrique Ribeiro Menezes ${ }^{1} \quad$ Alexandre Queiroz Bracarense ${ }^{1}$ \\ Recibido 15 de mayo de 2018, aceptado 25 de marzo de 2019 \\ Received: May 15, 2018 Accepted: March 25, 2019
}

\begin{abstract}
RESUMEN
La soldadura GMAW es un proceso enormemente versátil debido a la variedad de aleaciones soldables, espesuras, posición de soldadura, alta productividad, viabilidad en relación a los otros procesos, fácil mecanización y automatización. La técnica es caracterizada por sus modos de transferencia metálica, los cuales, tienen aplicaciones específicas en cuanto a la espesura del material base y la posición de soldadura. Por tanto, se hace necesaria una buena caracterización y conocimiento de esos modos. De esta manera, este trabajo es propuesto con el objetivo de identificar la transición entre los modos de transferencia globular y "spray" a través del desarrollo de una curva característica del proceso. Para eso, un primer abordaje del estudio consistió en la búsqueda de parámetros de soldadura deseados y posteriormente, mediante el uso de un sistema de recolección de gotas, fueron obtenidas gotas de varios test asociadas con las corrientes de soldadura utilizadas. Así, después de analizar y tratar los datos obtenidos, fue posible trazar una curva Volumen de gota vs Corriente de soldadura, la cual caracteriza la transferencia globular y spray, y la región de transición entre ellas.
\end{abstract}

Palabras clave: GMAW, transferencia metálica, corriente de transición.

\begin{abstract}
GMAW welding is an extremely versatile process due to the variety of weldable alloys, thicknesses, welding position, high productivity, viability compared to other processes, easy mechanization and automation. The technique is characterized by its metallic transfer modes, which have specific applications regarding material thickness and welding position. Therefore, the good characterization and knowledge of these modes is necessary. In this way, this work was proposed with the objective of identifying the transition between the globular and "spray" transfer modes through the development of a characteristic curve of the process. For this, a first approach of the study was done searching for the desired welding parameters and, later, through the use of a droplet collection system, droplets of several tests were obtained and associated with the welding currents used. Thus, after analyzing and processing the data obtained, it was possible to trace the Droplet Volume vs welding Current curve, that characterizes the Globular and Spray transfers and the transition region between them.
\end{abstract}

Keywords: GMAW, metal transfer, transition current.

\footnotetext{
1 Universidad Federal de Minas Gerais. Laboratório de Robótica, Soldagem e Simulação - LRSS. Programa de Ingeniería Mecánica. Belo Horizonte, Brasil.

E-mail: andresmauriciomu@ufmg.br; kkromanelli@yahoo.com.br; pedro_morada@hotmail.com; queiroz@demec.ufmg.br

* Autor de correspondencia: andresmauriciomu@ufmg.br
} 


\section{INTRODUCCIÓN}

Como la transferencia metálica ocurre es fundamental en la soldadura MIG/MAG, pues la influencia de muchas características del proceso, a saber: la estabilidad del arco eléctrico, la cantidad de gases absorbidos por el metal fundido, la posibilidad de aplicar la técnica en ciertas posiciones, geometría del cordón, etc. Además, el modo de transferencia depende de otros factores como el tipo y el valor de la corriente, tensión y polaridad, el diámetro del metal de adición, tipo y composición del medio de protección, el stick-out (longitud de alambre energizado), etc. [1].

Como consecuencia de la cantidad de variables que ejercen efecto sobre el proceso, la transferencia metálica en GMAW se puede dar de diversos modos. Sin embargo, se consideran tres formas principales: transferencia por Cortocircuito, Globular y Spray. Las regiones de tensión y corriente de estos tres modos de transferencia, con el uso de polaridad inversa (Configuración del electrodo en el polo positivo - DCEP) y gas de protección a base de Argón, se muestran cualitativamente en la Figura 1.

Con lo anterior, partiendo de la transferencia Globular e incrementando la corriente, el diámetro de las gotas disminuye hasta alcanzar la interfaz entre la transferencia globular y Spray, conocida

Fuente: Adaptado [2].

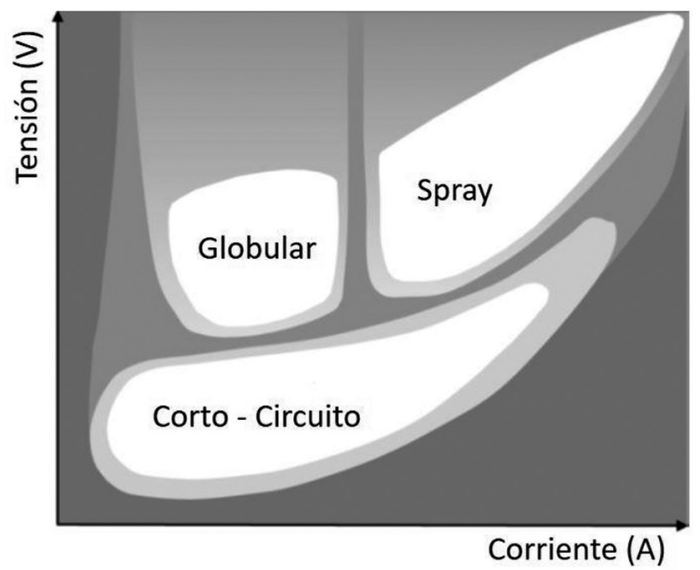

Figura 1. Condiciones de corriente y tensión para las diferentes formas de transferencia en GMAW con un gas de protección a base de Argón (esquemático). como "corriente de transición", la cual, marca la disminución abrupta del diámetro de las gotas y el incremento de la frecuencia de transferencia de las mismas, debido a la mudanza de fenómenos que gobiernan la deposición de metal [3]. La Figura 2 ilustra la ocurrencia de la corriente de transición, evidenciándose la variación repentina en el volumen de las gotas de metal líquido y su cantidad, para determinado rango de valores de corriente.

En la tarea de caracterizar la corriente de transición, técnicas experimentales han sido utilizadas en el estudio de la transferencia metálica. Métodos fotográficos o de filmación, han auxiliado en la visualización de los fenómenos, como en el caso del uso del oscilógrafo ultravioleta junto con grabación cinematográfica de alta velocidad [4]: sin embargo, se ha concluido que la medición de la frecuencia de destacamento en el modo spray es bastante difícil, así como también, la medición del diámetro de la gota debido a su irregularidad. Igualmente, la aplicación de métodos mecánicos para la recolección de gotas del metal de aporte, permite la evaluación del proceso [5].

Con todo, la clara definición entre las transferencias globular y spray, es la justificativa de este trabajo, en el cual, la identificación de la corriente de transición entre tales modos de deposición, es presentada a través de la curva que relaciona el volumen de la gota y la corriente.

Fuente: Adaptado [6].

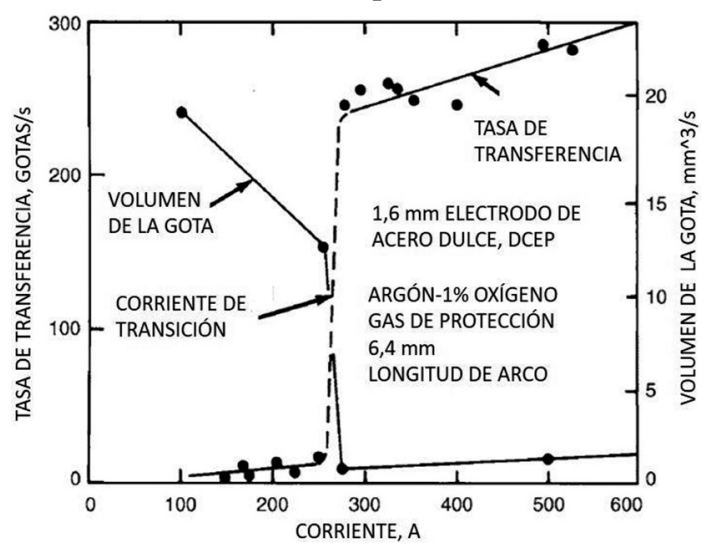

Figura 2. Variación en volumen y tasa de transferencia de gotas en función de la corriente de soldadura (electrodo de acero). 


\section{METODOLOGÍA}

\section{Materiales y Equipos}

Para el desarrollo de esta investigación, se utilizó una máquina de soldadura GMAW convencional (Fuente de alimentación de tensión constante y alimentador de alambre-electrodo con velocidad constante) marca ESAB, modelo Smashweld 408. Un consumible AWS ER70S- 6 con diámetro de $0,8 \mathrm{~mm}$ y un gas de protección con $2 \%$ de $\mathrm{O}_{2}$ y $98 \%$ de Ar. Para el análisis de las características eléctricas del arco (tensión y corriente), fue utilizado el sistema de adquisición de datos SAP V4 Ti de la marca IMC Soldagem, con una tasa de 5.000 medidas por segundo. Para el estudio de la transferencia metálica, fue adoptada una técnica mecánica fundamentada en el método presentado por Bracarense y Liu [7]; este procedimiento permite que las gotas puedan ser colectadas, medidas, pesadas y estudiadas, considerando también, que representa un montaje y una manipulación más simples en comparación con sistemas ópticos. De este modo, la recolección de las gotas fue hecha utilizando un sistema que consta de un tubo refrigerado por agua, instalado sobre un tanque de colecta y un sistema de refrigeración (tanque y bomba hidráulica sumergida), responsable por la circulación del agua dentro del tubo de cobre. La Figura 3 presenta el diseño esquemático del sistema de colecta de gotas.
En relación a los consumibles del proceso, la escogencia del gas de protección fue basada en trabajos que relatan la posibilidad de ocurrencia de trasferencia metálica del tipo spray [2] para un porcentaje de $2 \%$ Oxígeno. También, el flujo volumétrico utilizado fue de 12 I min-1, con lo cual, se garantizó una total protección del arco eléctrico como respuesta a las mayores corrientes usadas en el modo de transferencia spray.

En la selección del diámetro del alambre-electrodo, fue considerado el factor limitante del espesor de la pared del tubo de cobre. Es conocido [8], que alambres-electrodo de mayor diámetro requieren una más alta corriente mínima de fusión en comparación con un electrodo más fino, para el mismo tipo de transferencia metálica. Por tanto, la selección del consumible de $0,8 \mathrm{~mm}$, se encuentra entre los menores valores de corriente de transición relatados [9], representando así en un menor aporte térmico y la garantía de preservación del tubo de cobre.

\section{Consideraciones iniciales}

Con el objetivo de garantizar puntos de comparación entre los diferentes ensayos, se definió el tiempo de soldadura en aproximadamente 15 segundos, contados a partir de la abertura del arco eléctrico. Además, los ensayos fueron realizados manualmente, con el auxilio de un soldador certificado. De este modo,

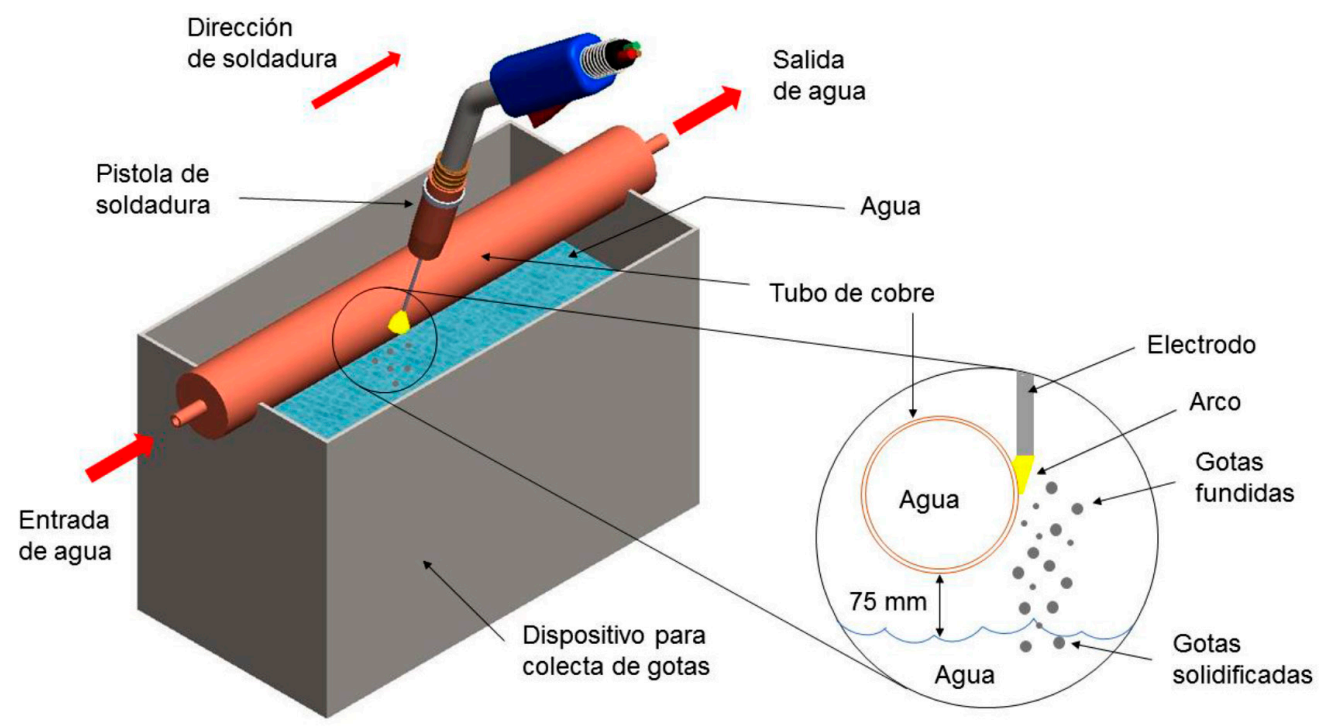

Figura 3. Diseño esquemático del sistema de colecta de gotas. Este sistema se basa en el método utilizado por [7]. 
se torna difícil mantener constante la longitud de alambre energizado y la velocidad de desplazamiento de la pistola de soldadura; por lo cual, estos son limitados a la destreza y capacidad del operador, quien utilizó un punto de apoyo en conjunto con una postura y conciencia corporal adquiridas con la experiencia. Con todo, fue utilizado un stick-out promedio de $15 \mathrm{~mm}$ y la velocidad de soldadura fue de $0,6 \mathrm{~m} \mathrm{~min}^{-1}$ en promedio.

En relación con el posicionamiento de la antorcha de soldadura, se garantizó que el alambre fuese tangente al tubo de cobre, como mostrado en el detalle de la Figura 3, de modo a que las gotas de metal líquido transferidas desde la punta del electrodo, hicieran contacto con el tubo refrigerado y cayeran en el colector con agua, asegurando de este modo, la solidificación del material de aporte e impidiendo su aglutinación.

\section{Procedimiento Experimental}

Inicialmente y utilizando una placa de acero ASTM A-36, fueron llevadas a cabo pruebas cualitativas con el propósito de determinar los menores valores de tensión en los cuales, es posible obtener los modos de transferencia Globular y Spray. Así, partiendo desde una configuración de tensión y velocidad de alimentación en la cual se logra la transferencia spray (Punto 1, Figura 4), se mapearon sobre la primera, valores para la velocidad de alimentación del electrodo (punto 2, 3 y 4), hasta el punto de obtener la transferencia tipo globular. De este modo, este procedimiento continúa como presentado en la Figura 4, reduciendo la tensión de operación y realizando un mapeo entre los dos modos de transferencia y la corriente de transición entre ellos. Por último, con la obtención de transferencia por corto-circuito para un determinado valor de tensión, es escogida una tensión anterior a esa y se define como el nivel válido para el desarrollo del estudio.

Con la confirmación de la posibilidad de obtención de los dos modos de transferencia por medio del sistema de adquisición de datos, fueron extrapolados esos ajustes para la aplicación en el colector de gotas. De esta manera, la tensión es mantenida constante y se varía apenas la velocidad de alimentación del electrodo para cada ensayo.

Luego de cada prueba, las gotas fueron debidamente recogidas utilizando un imán. Enseguida, estas
Fuente: Adaptado [6].

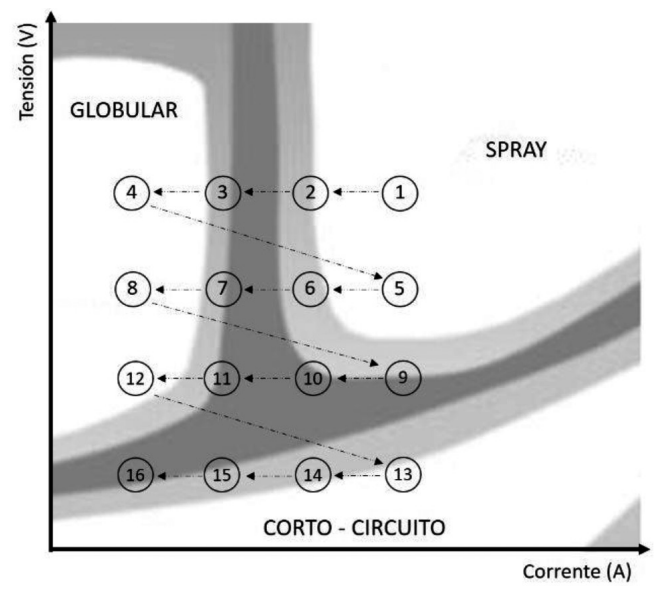

Figura 4. Esquema del procedimiento aplicado en las pruebas preliminares para la definición del valor de tensión. Técnica de cordón sobre chapa, electrodo AWS ER 70S-6 de $0,8 \mathrm{~mm}$, stick-out de $15 \mathrm{~mm}$, velocidad de soldadura de $0.6 \mathrm{~m}$ min-1, protección: Ar-2\%O2.

eran secadas para evitar la oxidación durante el almacenamiento y pesadas utilizando una balanza de precisión. Al terminar las soldaduras en el sistema de colecta, se dio inicio a la etapa de clasificación de las gotas por diámetro. Las muestras fueron separadas utilizando tamices granulométricos, comúnmente empleados en el tratamiento de minerales. En total fueron 7 tamices, obteniéndose 8 niveles de estratificación de las muestras ( 7 partes retenidas en los tamices y 1 pasante en la más fina) que fueron pesadas separadamente. Los tamices utilizados y sus características están listados (en orden decreciente de abertura) en la Tabla 1.

Tabla 1. Tamices utilizados y sus aberturas.

\begin{tabular}{|c|c|c|}
\hline \multicolumn{2}{|c|}{ Seri } & \multirow{2}{*}{ Abertura (mm) } \\
\cline { 1 - 2 } ASTM & Tyler & \\
\hline 8 & 8 & 2,36 \\
\hline 12 & 10 & 1,70 \\
\hline 16 & 14 & 1,18 \\
\hline 18 & 16 & 1,00 \\
\hline 20 & 20 & 0,85 \\
\hline 30 & 28 & 0,60 \\
\hline 35 & 32 & 0,50 \\
\hline
\end{tabular}


Con la masa de las gotas retenidas en cada tamiz y las pasantes para el último estrato, se realizó una normalización en función de la masa total de cada muestra. Lo anterior, se llevó a cabo con el fin lograr la comparación directa entre las diferentes muestras, ya que la variación de la velocidad de alimentación del metal de adición, implica en la mudanza de la cantidad de material depositado, resultando en masas diferentes para cada ensayo. Fue obtenida así, la masa relativa, la cual es el resultado de la división entre la masa retenida en cada tamiz y la masa de la muestra total de cada ensayo.

De este modo, con el valor de la masa relativa, es posible determinar el diámetro característico de cada muestra. Dos métodos fueron utilizados en esta tarea: el primero, Criterio d50 [10], que consiste básicamente de una interpolación lineal utilizando como puntos conocidos, las dimensiones de las aberturas de los tamices asociados a las masas relativas acumuladas en los mismos, entre las cuales está el valor de masa acumulada de $50 \%$, resultando en el diámetro característico d50.

Para el segundo método de cálculo del diámetro característico de las muestras, basado en [11], fue desarrollada una media ponderada, la cual se obtiene multiplicando el promedio simple entre la dimensión de abertura de un par de tamices (Tabla 2) por su ponderación (masa retenida por tamiz en gramos), para luego dividir esta suma ponderada entre la sumatoria de los pesos. La ecuación 1 demuestra el cálculo del diámetro característico ponderado, dpond.

Tabla 2. Media entre la abertura de los tamices.

\begin{tabular}{|c|c|c|}
\hline Tyler & $\begin{array}{c}\text { Abertura } \\
(\mathbf{m m})\end{array}$ & $\begin{array}{c}\text { Media entre } \\
\text { aberturas (mm) }\end{array}$ \\
\hline 7 & 2,80 & - \\
\hline 8 & 2,36 & 2,58 \\
\hline 12 & 1,70 & 2,03 \\
\hline 16 & 1,18 & 1,44 \\
\hline 18 & 1,00 & 1,09 \\
\hline 20 & 0,85 & 0,92 \\
\hline 30 & 0,60 & 0,72 \\
\hline 35 & 0,50 & 0,55 \\
\hline Fondo $^{1}$ & 0,00 & 0,25 \\
\hline
\end{tabular}

1 Fondo: último nivel de estratificación.

$$
d_{\text {Pond }}=\frac{\sum_{i=1}^{n} m i R i}{\sum_{i=1}^{n} m i}
$$

Donde:

mi: valor promedio de la abertura del tamiz i;

Ri: masa retenida en el tamiz i;

Con la finalidad de comparar los resultados de esta investigación con lo expuesto en la literatura [6, $3,4]$, la cual siempre se relaciona la frecuencia de transferencia de gotas vs la corriente o el volumen de la gota vs la corriente, se calculó el volumen característico de las gotas de 11 muestras. Para eso, se aplica la metodología inversa desarrollada por [11], quien primero determinó el volumen y luego, el diámetro característico. Así, asumiendo que las gotas transferidas del alambre-electrodo responden a una geometría esférica, por medio de la Ec. 2 es calculado el volumen característico V.

$$
V=\frac{\pi d^{3}}{6}
$$

Donde:

d: diámetro medio característico;

También, las señales adquiridas en cada test fueron relacionadas con las gotas obtenidas en el mismo. A partir del análisis anterior, se determinó la corriente de operación de cada soldadura para así, asociarla con el volumen característico y obtener la curva de esas grandezas. Los datos de tensión y corriente media fueron obtenidos mediante el tratamiento de señales con el uso de Sinal 2017, software desarrollado en el Laboratorio de Robótica, Soldadura y Simulación - LRSS de la Escuela de Ingeniería de la UFMG.

\section{RESULTADOS Y DISCUSIONES}

\section{Pruebas preliminares}

Con el sistema de adquisición de datos enlazado al equipo de soldadura, fueron realizadas pruebas para una tensión en torno de $26 \mathrm{~V}$, variando la velocidad de alimentación y buscando identificar las trasferencias Globular, Spray y la transición entre ellas. De este modo, por medio del estudio de los oscilogramas de tensión y corriente, fue corroborado el comportamiento característico de cada tipo de 
transferencia observado. Para el valor de tensión anterior, el comportamiento de los oscilogramas fue clasificado dentro de los modos de transferencia de estudio; sin embargo, con la finalidad de disminuir el aporte térmico y así preservar el tubo de cobre, se da continuidad al procedimiento de la Figura 4. Por tanto, se disminuyó la tensión hasta un valor de $25 \mathrm{~V}$ y fueron repetidas las pruebas mediante la corroboración de las señales eléctricas del arco, dando como resultado otro posible valor de aplicación para el estudio. Nuevamente, el proceso continua hasta una tensión de $23 \mathrm{~V}$, donde ocurre transferencia por Corto-circuito, por lo cual se retornó a la última configuración exitosa. Así, para una tensión de operación de $24 \mathrm{~V}$, fue posible identificar los fenómenos de transferencia en estudio y además, para este ajuste en conjunto con una velocidad de alimentación de alambre-electrodo de 10,5 $\mathrm{m} \mathrm{min}^{-1}$, se obtuvo un modo de transferencia entre la región de transición Globular-Spray.

\section{Pruebas en el colector de gotas}

El ajuste de $24 \mathrm{~V}$ y $10,5 \mathrm{~m} \mathrm{~min}^{-1}$ de velocidad de alimentación de alambre, fue transferido directamente para el tubo de cobre, con el intuito de obtener la región de transición. A pesar de creer que habría necesidad de reajustar estos parámetros para uso en cobre, la configuración de estas variables se mostró adecuada; en otras palabras, el cobre (diferente del acero al carbono), presenta dificultades importantes cuando requerido para unión de materiales: su alta conductividad térmica representa un desafío al momento de soldar, por lo cual no se conoce aplicación directa de ese proceso de soldadura, adoptando técnicas de unión como "brazing" o "soldering", en función del punto de fusión del metal de adición. Considerando lo anterior, en el desarrollo de este proyecto, se esperaba que al cambiar el "metal base" de los ensayos, los parámetros de operación establecidos en la chapa de acero A-36, no tuvieran los mismos resultados para la aplicación en el colector de gotas, por el hecho de que se tiene un tubo de cobre, refrigerado a agua, que se pensaba iba a reaccionar diferente al establecimiento del arco eléctrico. Finalmente, cuando fueron realizados los ensayos, se constató el mismo comportamiento del arco eléctrico y los tipos de transferencia metálica en el cobre.

Paso seguido, se modificó la velocidad de alimentación del electrodo hasta $7 \mathrm{~m} \mathrm{~min}^{-1}$, pudiéndose observar una transferencia del tipo Globular bien definida y característica, sustentada por la evaluación de señales y la colecta de gotas.

Posteriormente, se mudó el ajuste de la velocidad de alimentación del alambre-electrodo, con el objetivo de obtener una mayor cantidad de datos y así, enriquecer el levantamiento de la curva para la región de transición. No obstante, fue posible aumentar la configuración de la velocidad de alimentación del alambre-electrodo hasta $15,5 \mathrm{~m} \mathrm{~min}^{-1}$, punto en el cual el tubo de cobre fue averiado. Este fenómeno es una respuesta directa del aumento de la velocidad de soldadura, lo cual requiere de la fuente eléctrica mayores niveles de corrientes, derivando en un aporte térmico considerable para el proceso. Fueron realizados 11 ensayos, con velocidades de alimentación de 7, 8, 8,5, 9, 9,5, $10,5,11,12,12,5,13,5, y 15,5 \mathrm{~m} \mathrm{~min}^{-1}$. En total, fueron realizadas 3 repeticiones para cada uno de los ensayos anteriores, menos para la velocidad de $15,5 \mathrm{~m} \mathrm{~min}^{-1}$, en la cual se fundió el tubo de cobre en la primera tentativa.

\section{Colecta y clasificación de las gotas}

Durante la realización de los experimentos, la diferencia entre el tamaño de las gotas para cada ensayo fue evidente. Para la trasferencia Globular por ejemplo, se observó una predominante cantidad de gotas grandes y hasta eventualmente, la ausencia de gotas pequeñas. Sobre la región de transición, se percibió claramente la disminución en el tamaño de las gotas y todavía, la ocurrencia de gotas mayores. Ya para la transferencia Spray, gotas diminutas y en gran cantidad fueron el resultado. En definitiva, el comportamiento previsto fue muy evidente: a medida que se aumentó la corriente de operación (velocidad de alimentación del alambre-electrodo), incrementó el número de gotas y disminuyó el diámetro de las mismas, como resultado de la mudanza de los fenómenos que gobiernan cada tipo de trasferencia. Las gotas colectadas para cada ensayo son presentadas en la Figura 5.

Además, en la Figura 6 es presentada la clasificación granulométrica para dos ajustes en la velocidad de alimentación del alambre y un tiempo de 15 segundos de soldadura. Se puede evidenciar que este parámetro tiene una relación directamente proporcional con la cantidad y tamaño de las gotas de metal transferido, como antes mencionado. 


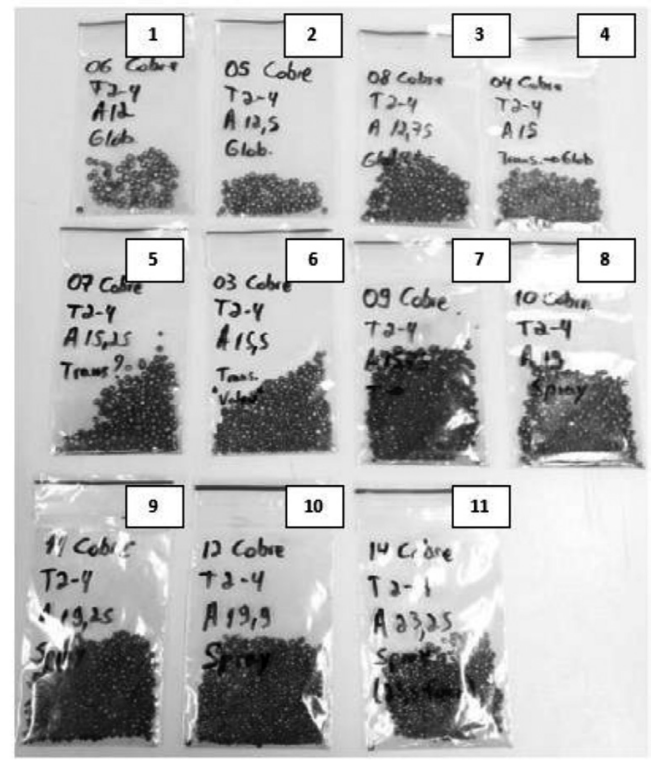

Figura 5. Gotas colectadas para cada valor de velocidad de alimentación de alambreelectrodo. $1.7 \mathrm{~m} \mathrm{~min}^{-1} ; 2.8 \mathrm{~m} \mathrm{~min}^{-1}$; 3. $8,5 \mathrm{~m} \mathrm{~min}^{-1} ; 4.9 \mathrm{~m} \mathrm{~min}^{-1} ; 5.9,5 \mathrm{~m} \mathrm{~min}^{-1}$; 6. $10,5 \mathrm{~m} \mathrm{~min}^{-1} ; 7.11 \mathrm{~m} \mathrm{~min}^{-1}$; 8. $12 \mathrm{~m} \mathrm{~min}^{-1}$; 9. $12,5 \mathrm{~m} \mathrm{~min}^{-1}$; 10. $13,5 \mathrm{~m} \mathrm{~min}^{-1}$; $11.15,5 \mathrm{~m} \mathrm{~min}^{-1}$.
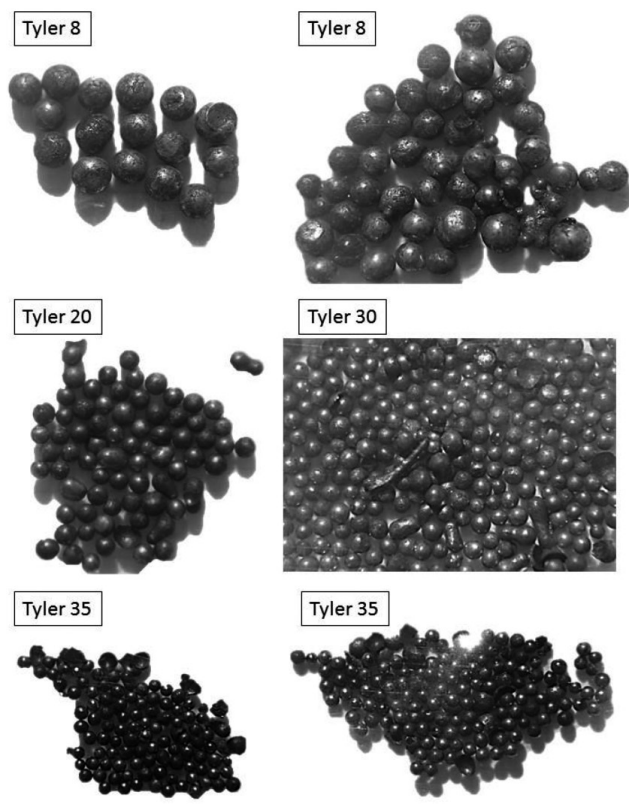

Figura 6. Clasificación granulométrica por Serie Tyler. Columna izquierda: gotas resultantes para $10,5 \mathrm{~m} \mathrm{~min}^{-1}$. Columna derecha: velocidad de alimentación de $12,5 \mathrm{~m} \mathrm{~min}^{-1}$.
El resultado del uso de los criterios d50 y dpond es presentado en la Tabla 3. Es posible evidenciar que los valores de diámetros característicos por las dos técnicas, permiten suponer que los resultados son bastante próximos, en función de sus diferencias (d).

Tabla 3. Diámetro característico.

\begin{tabular}{|c|c|c|c|}
\hline $\begin{array}{c}\text { Velocidad de } \\
\text { Alimentación } \\
\left(\mathbf{m} \mathbf{m i n}^{-1}\right)\end{array}$ & $\begin{array}{c}\mathbf{d 5 0} \\
(\mathbf{m m})\end{array}$ & $\begin{array}{c}\text { dpond } \\
(\mathbf{m m})\end{array}$ & $\begin{array}{c}\mathbf{d} \\
(\mathbf{d p o n d}- \\
\mathbf{d 5 0}(\mathbf{m m}) \mathbf{)}\end{array}$ \\
\hline 7 & 2,525 & 2,455 & $-0,070$ \\
\hline 8 & 2,189 & 2,171 & $-0,018$ \\
\hline 8,5 & 2,143 & 2,099 & $-0,044$ \\
\hline 9 & 2,099 & 2,063 & $-0,036$ \\
\hline 9,5 & 2,07 & 2,026 & $-0,044$ \\
\hline 10,5 & 1,749 & 1,709 & $-0,040$ \\
\hline 11 & 1,898 & 1,851 & $-0,046$ \\
\hline 12 & 1,849 & 1,795 & $-0,054$ \\
\hline 12,5 & 1,321 & 1,4 & 0,079 \\
\hline 13,5 & 1,209 & 1,342 & 0,133 \\
\hline 15,5 & 1,197 & 1,276 & 0,080 \\
\hline
\end{tabular}

Con el objetivo de probar la hipótesis estadística de que los valores medios de los diámetros característicos d50 y dpond ( $\mu_{1}$ y $\mu_{2}$, respectivamente) responden al enunciado $\mathrm{H}_{0}: \mu_{1}=\mu_{2}$, se realizó una prueba T pareada. Según Montgomery [12] en este tipo de comparación, los niveles del factor están "pareados" en cada unidad experimental, el cual es el caso de este estudio. De este modo, utilizando un nivel de significancia $(\alpha)$ de 0,05 , la hipótesis nula $\left(\mathrm{H}_{0}\right)$ considerada fue que la media de cada parámetro analizado d50 fuera igual a dpond. Como hipótesis alternativa $\left(\mathrm{H}_{1}\right)$ fue considerado que el promedio de los diámetros característicos es diferente; las ecuaciones Ec. 3 y Ec. 4 se refieren a la hipótesis nula y alternativa, respectivamente. Las principales respuestas de la prueba para cada velocidad de alimentación pueden ser verificadas en la Tabla 4.

$$
\begin{aligned}
& H_{0}: \mu_{d}=0 \\
& H_{1}: \mu d \neq 0
\end{aligned}
$$

De este modo, se comparó el valor calculado del estadístico de prueba t pareado con la región crítica $\pm \mathrm{t}_{0,025,5}=2,571$. Así, como $\left|\mathrm{t}_{0}\right|=1 \ngtr \mathrm{t}_{0,025,5}=2,571$, 
Tabla 4. Valores de la prueba $\mathrm{T}$ pareada para los ensayos realizados.

\begin{tabular}{|c|r|c|r|}
\hline $\begin{array}{c}\text { Velocidad de } \\
\text { Alimentación } \\
\left(\mathbf{m} \mathbf{~ m i n}^{-1}\right)\end{array}$ & $\overline{\mathrm{d}}^{2}$ & $\mathbf{S}_{\mathbf{d}}^{\mathbf{3}}$ & $\mathbf{t}_{\mathbf{0}}{ }^{\mathbf{4}}$ \\
\hline 7 & 0,01167 & 0,00476 & 1 \\
\hline 8 & 0,00300 & 0,00122 & 1 \\
\hline 8,5 & 0,00733 & 0,00299 & 1 \\
\hline 9 & 0,00600 & 0,00245 & 1 \\
\hline 9,5 & 0,00733 & 0,00299 & 1 \\
\hline 10,5 & 0,00667 & 0,00272 & 1 \\
\hline 11 & 0,00783 & 0,00320 & 1 \\
\hline 12 & 0,00900 & 0,00367 & 1 \\
\hline 12,5 & $-0,01317$ & 0,00538 & -1 \\
\hline 13,5 & $-0,02217$ & 0,00905 & -1 \\
\hline 15,5 & $-0,01317$ & 0,00538 & -1 \\
\hline
\end{tabular}

la hipótesis no puede rechazarse. En otras palabras, para el nivel de significancia de 5\%, no hay evidencia que indique que $\mathrm{d} 50 \mathrm{y}$ dpond son diferentes.

Lo anterior posiblemente justifica la utilidad de la metodología, una vez que los resultados se aproximan a un método validado [10].

Asimismo, el volumen característico obtenido mediante el uso de los dos criterios, junto con diferencia de Vdpond en relación a Vd50, es presentado en la Tabla 5.

También, es importante indicar la presencia de defectos en las gotas recolectadas para los diferentes

Tabla 5. Volumen característico.

\begin{tabular}{|c|c|c|c|}
\hline $\begin{array}{l}\text { Velocidad de } \\
\text { Alimentación } \\
\left(\mathrm{m} \mathrm{min} \mathbf{m}^{-1}\right)\end{array}$ & $\begin{array}{l}\text { Vd50 } \\
\left(\mathbf{m m}^{3}\right)\end{array}$ & $\begin{array}{c}\text { Vdpond } \\
\left(\mathbf{m m}^{3}\right)\end{array}$ & $\begin{array}{l}\text { Vdpond } \\
\text { - Vd50 } \\
\left(\mathrm{mm}^{3}\right)\end{array}$ \\
\hline $\mathrm{mm}$ & 8,427 & 7,747 & $-0,679$ \\
\hline 8 & 5,492 & 5,358 & $-0,134$ \\
\hline 8,5 & 5,153 & 4,84 & $-0,314$ \\
\hline 9 & 4,841 & 4,595 & $-0,246$ \\
\hline 9,5 & 4,647 & 4,357 & $-0,290$ \\
\hline 10,5 & 2,802 & 2,613 & $-0,189$ \\
\hline 11 & 3,58 & 3,323 & $-0,256$ \\
\hline 12 & 3,31 & 3,03 & $-0,280$ \\
\hline 12,5 & 1,207 & 1,438 & 0,231 \\
\hline 13,5 & 0,925 & 1,265 & 0,340 \\
\hline 15,5 & 0,897 & 1,089 & 0,192 \\
\hline
\end{tabular}

ensayos. En algunos casos, la presencia de gotas aglutinadas (el cual es el principal cuestionamiento de esta técnica [5]) llevó a la depuración de la totalidad de la muestra y una reclasificación en los tamices granulométricos. La Figura 7 presenta el defecto por aglutinamiento en gotas retenidas en tamiz de la serie Tyler 8 .

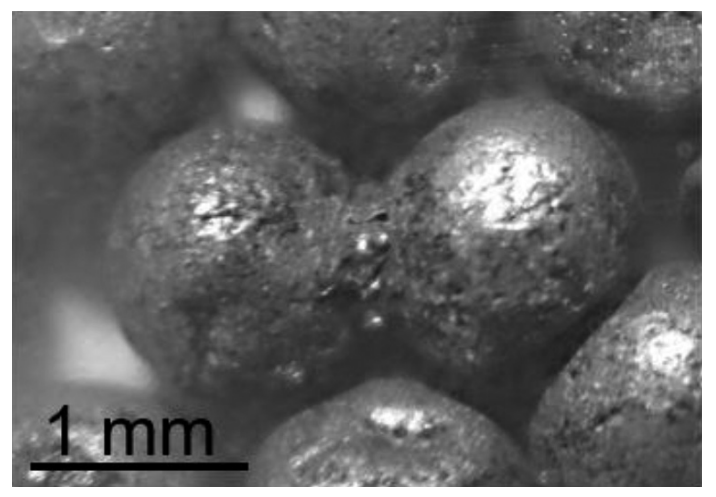

Figura 7. Gotas aglutinadas.

\section{Análisis de las señales}

Los datos obtenidos de los 11 test fueron tratados y trazados por medio de oscilogramas de las dos principales características eléctricas del arco en función del tiempo: tensión y corriente. La Tabla 6 muestra las medias de los valores de corriente y la dispersión estadística de las pruebas.

También, Ciclogramas presentando la dispersión de los valores de tensión y corriente para cada ensayo, fueron realizados con el objetivo de acompañar la identificación del tipo de transferencia metálica. De este modo, es posible observar en la Figura 8, para una velocidad de alimentación de $7 \mathrm{~m}$ min-1, la nube de puntos que representa un típico modo Globular. Asimismo, la transferencia spray (Figura 10), resultante de una velocidad de alimentación asignada en $13,5 \mathrm{~m}$ min-1, exhibe un comportamiento estable, caracterizado por el agrupamiento de la nube de puntos para altos valores de corriente y sin notables dispersiones de los mismos. La mayor dispersión de los valores de corriente y tensión durante el modo globular en relación con spray, se debe posiblemente a la variación de la longitud del arco eléctrico durante el crecimiento de las gotas en la punta del alambre-electrodo, y su traslado por el arco. En la región de transición (Figura 9), se 
Tabla 6. Corriente media y desviación estándar en función de la variación de la velocidad de alimentación del alambre-electrodo.

\begin{tabular}{|c|c|c|}
\hline Velocidad de Alimentación $\left(\mathbf{m ~ m i n}^{\mathbf{- 1}} \mathbf{)}\right.$ & Corriente Media (A) & Desvío de la Corriente Media (A) \\
\hline 7 & 80,19 & 57,71 \\
\hline 8 & 97,26 & 54,79 \\
\hline 8,5 & 125,20 & 36,69 \\
\hline 9 & 133,19 & 41,76 \\
\hline 9,5 & 131,46 & 37,40 \\
\hline 10,5 & 131,97 & 62,60 \\
\hline 11 & 139,62 & 37,93 \\
\hline 12 & 138,97 & 52,97 \\
\hline 12,5 & 136,26 & 46,40 \\
\hline 13,5 & 145,11 & 50,91 \\
\hline 15,5 & 160,57 & 5,78 \\
\hline
\end{tabular}

observan puntos dispersos que reflejan inestabilidad durante las pruebas, generados probablemente por breves comportamientos de mudanza del modo de transferencia (entre globular y spray) [2] y hasta una aparente extinción momentánea del arco. De modo general, para las regiones de transferencia Globular y de corriente de transición, se pueden asociar en parte las mayores fluctuaciones de tensión y corriente con el crecimiento y transferencia de las gotas.

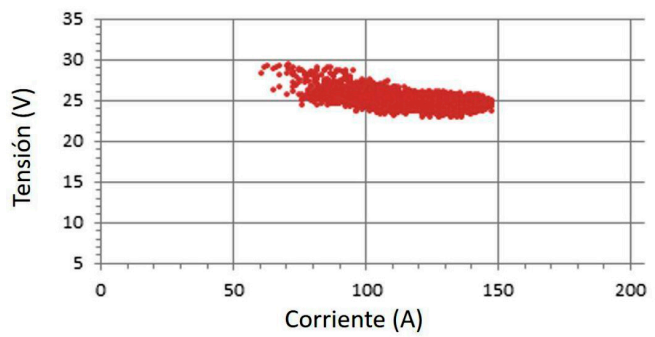

Figura 8. Ciclograma de corriente vs tensión para transferencia Globular. Velocidad de alimentación de $7 \mathrm{~m} \mathrm{~min}^{-1}$.

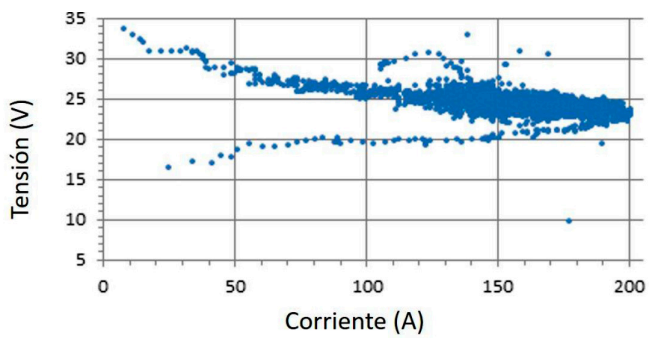

Figura 9. Ciclograma de corriente vs tensión para transferencia "Corriente de transición". Velocidad de alimentación de $10,5 \mathrm{~m} \mathrm{~min}^{-1}$.

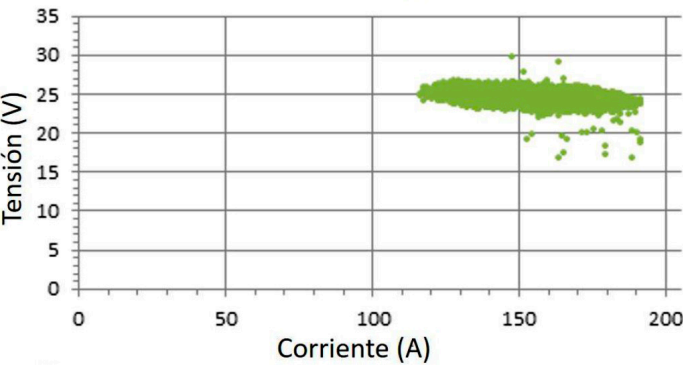

Figura 10. Ciclograma de corriente vs tensión para transferencia Spray. Velocidad de alimentación de 13,5 $\mathrm{m} \mathrm{min}^{-1}$.

Con base en los ciclogramas presentados anteriormente y a modo de comparación con la Figura 2, fue posible graficar una distribución corriente vs tensión utilizando regiones de estabilidad de los ajustes en velocidad de alimentación para los 11 ensayos. Ese gráfico contiene un total de 825.000 puntos, que hacen referencia a 5 segundos de adquisición de datos. El gráfico fue ploteado como 11 conjuntos de nubes de puntos, representado la transición de globular a Spray.

Como se observa en la Figura 11, el modo de transferencia Globular se encuentra bien definido, situado a la izquierda del gráfico: la región exhibe la particularidad de una baja corriente y alta tensión, como resultado de los fenómenos que ocurren durante la transferencia del metal de adición. Por su parte, la transferencia spray se halla a la derecha del gráfico, determinada por una mayor corriente de operación. Hacia el centro del gráfico, se encuentra la 


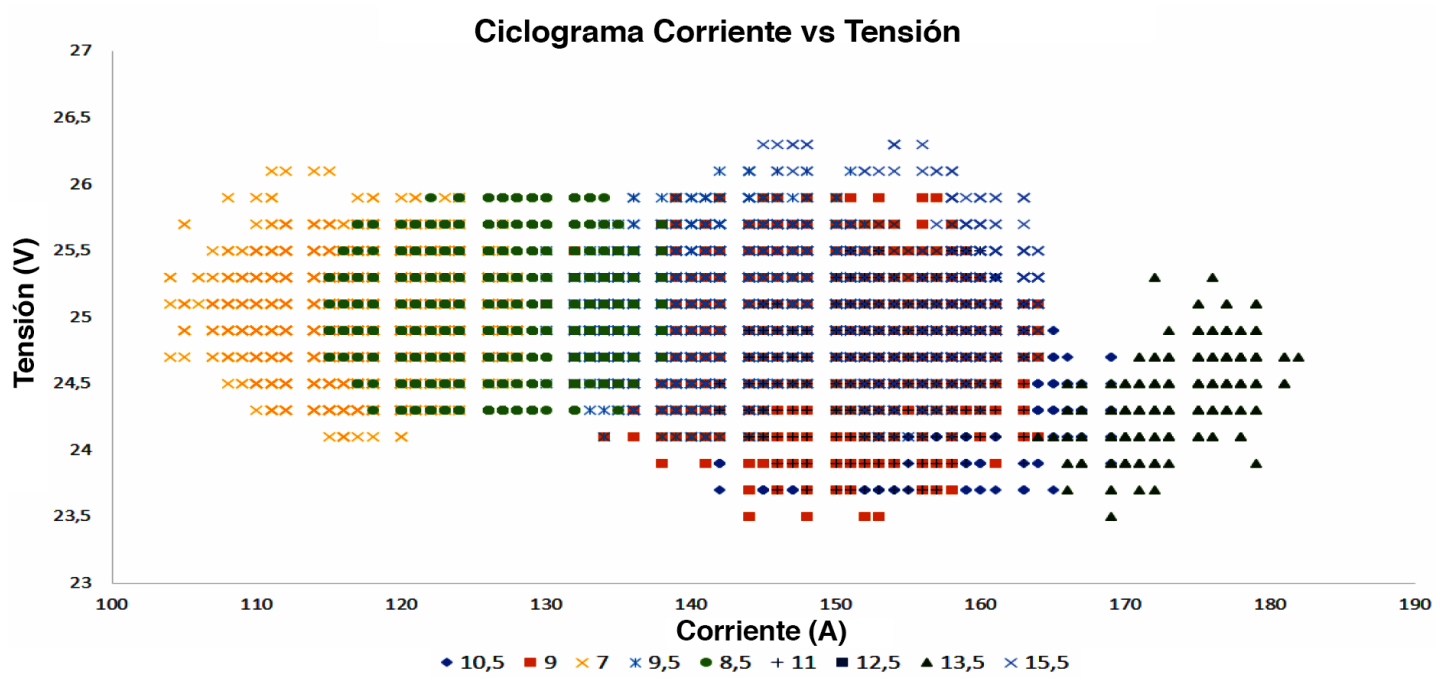

Figura 11. Ciclograma de corriente vs tensión para las regiones de estabilidad de los 11 ensayos.

interfaz de "corriente de transición", evidenciándose de esta manera la alternancia de periodos entre la transferencia Globular y Spray.

\section{RESULTADO FINAL}

Vinculando los datos obtenidos del Volumen de gota y su corriente media asociada, fue desarrollado el gráfico que caracteriza las transferencias metálicas Globular, Spray y la interfaz de corriente de transición, conforme presentado en la Figura 12. La comparación entre los dos métodos utilizados para obtener el volumen crítico está presentada en el gráfico. Las dos líneas muestran un comportamiento semejante (dpond y d50). Es posible evidenciar por ejemplo, como para una corriente de $130 \mathrm{~A}$, se da inicio a una franja entre las dos líneas paralelas, que se define como corriente de transición (entre

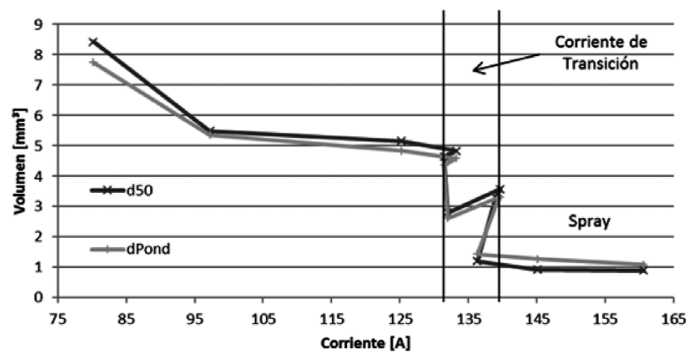

Figura 12. Variación del Volumen de las gotas con la corriente de soldadura.
130 y $140 \mathrm{~A}$ ) para un electrodo de $0,8 \mathrm{~mm}$, un gas de protección con $2 \%$ de $\mathrm{O} 2$ y $98 \%$ de $\mathrm{Ar}$ y una fuente a tensión constante.

\section{CONCLUSIONES}

Este artículo presenta datos experimentales sobre el comportamiento de la interfaz de corriente de transición entre los tipos de transferencia Globular y Spray. De este modo, los datos presentados resumen la metodología utilizada en el estudio de ese fenómeno, bajo la aplicación de técnicas mecánicas (sistema colector de gotas) y métodos indirectos (comprendiendo la medición de señales eléctricas relacionadas al proceso).

De acuerdo con los resultados de esta investigación, las principales conclusiones se presentan a continuación:

1. El cálculo del diámetro característico de la gota mediante el uso de una media ponderada (dpond), resultó en valores semejantes a los expresados mediante el criterio d50. Lo anterior, fue validado mediante una prueba t pareada, la cual estipula que no hay evidencias para aceptar una hipótesis en la cual dpond sea diferente de d50. Finalmente, estas dos técnicas permiten un cálculo simple para la obtención del volumen característico de gotas.

2. A pesar de la dificultad de garantizar que con el uso del sistema de colecta, las gotas de metal 
no se aglutinen antes de solidificar, el volumen característico resultante de la aplicación de este técnica mecánica, está en conformidad con lo expuesto en literatura [1].

3. El desarrollo de ciclogramas como método de evaluación del tipo de trasferencia metálica, representa el mejor modo de integrar la dispersión de valores de tensión y corriente de diferentes pruebas, suministrando un mejor y más rápido entendimiento de las variables en estudio. Además, el ciclograma de la Figura 11, reemplaza los clásicos esquemas que definen los modos de transferencia metálica para el proceso GMAW.

4. A partir del gráfico obtenido en esta investigación, se da un primer acercamiento hacia un verdadero entendimiento de los fenómenos gobernantes de la interfaz entre la transferencia del tipo Globular y Spray. Como se puede evidenciar en la Figura 12, la región de corriente de transición no responde a un comportamiento lineal, por lo que se puede formular una hipótesis, en la cual la alternancia de periodos entre los dos tipos de transferencias (Globular y Spray), se cree es gobernada por fuerzas aún no definidas (magnéticas, de tensión superficial, o del tipo gravitatorias).

5. Aunque las soldaduras fueron realizadas de forma manual, este estudio exploratorio suministra los parámetros necesarios para desarrollar una investigación con el uso de sistemas mecanizados y/o robotizados, aprobando así, la aplicación de esta metodología de análisis en proyectos de evaluación de transferencia metálica.

6. Se puede afirmar que el comportamiento general de las tres regiones de interés, está bien definido en el gráfico; incluso con los datos de la región central presentando una mayor variación entre sí. La zona ubicada en la parte superior izquierda, presenta una consistencia en relación con el alto volumen de gota (con diámetros mayores que el diámetro del alambre-electrodo, como mostrado en la Tabla 3), semejante al evidenciado en la Figura 1 (región Globular). Así, se evidencia la típica transferencia globular, caracterizada por baja corriente y gotas grandes. Por otro lado, la transferencia Spray, sobre la zona inferior derecha del gráfico (a la derecha de la región de transición), presenta una tendencia lineal entre el volumen de gota y la corriente; la Figura 2 constata el mismo comportamiento aquí relatado.

7. Nuevamente, entre esas dos regiones, es posible identificar el comportamiento específico de la interfaz de corriente de transición, el cual varía intermitentemente entre los dos modos de transferencia. A diferencia de los gráficos expuestos en la literatura [1-6], la interfaz objeto de estudio de esta investigación no presenta un comportamiento idealizado, ya que es reflejo de experimentación.

\section{AGRADECIMIENTOS}

Los autores agradecen al Laboratorio de Robótica, Soldagem e Simulação (LRSS) de la Universidad Federal de Minas Gerais.

\section{REFERENCIAS}

[1] A. Quites. "Introdução à Soldagem a Arco Voltaico". Soldasoft. 1 Edición. Florianópolis: Brasil, p. 352. 2002. ISBN: 85-89445-01-1.

[2] P. Modenesi. "Introdução à Física do Arco Elétrico E sua Aplicação na Soldagem dos Metais". Belo Horizonte: Brasil, p. 187. 2017.

[3] A. Scotti and V. Ponomarev. "Soldagem MIG/ MAG”. Artliber Editora Ltda. 1 Edición. São Paulo: BBrasil, p. 284. 2008.

[4] S. Rhee and E. Kannatey-Asibu. "Observation of Metal Transfer during Gas Metal Arc Welding". Welding Research Supplement - Welding Journal, pp. 381-386. October, 1992. ISSN: 0043-2296

[5] P. Modenesi. "Tranferência de Metal de Adição na Soldagem com Eletrodos Revestidos". Tecnologia de Soldagem. Vitória, Brasil. 1983.

[6] American Welding Society. "Welding Handbook Welding Processes, Part 1". Annette O'Brien - Editor. 9 Edición: Canadá, p. 788. 2004. ISBN: 0-87171-729-8

[7] A. Bracarense and S. Liu. "Chemical composition variations in shielded metal arc welds". Welding Research SupplementWelding Journal, pp. 529-536. December, 1993. ISSN: 0043-2296

[8] E. Wainer, S. Brandi and F. Décourt. "Soldagem Processos e Metalurgia". Blucher. 1 Edición - 6 Impresión. São Paulo: Brasil, p. 494. 2008. ISBN: 85-212-0238-5 
[9] ASM International. "Metals Handbook Welding Brazing and Soldering". ASM Handbook Committee. Vol. 6, p. 2873. Estados Unidos. 1993. ISBN: 0-87170-377-7 (V.1)

[10] J.H. Chen, D. Fan, Z.Q. YE and J. Luo. "A study of the mechanism for globular metal transfer from covered electrodes". Welding Journal, pp. 145-150. 1989. ISSN: 0043-2296
[11] A. Bracarense, R. Lacerda de Souza, M.C.M. de Souza Costa, P.E. Faria and S. Liu. "Welding current effect on diffusible hydrogen content in flux cored arc weld metal". Journal of the Brazilian Society of Mechanical Sciences. 2002. ISSN: 0100-7386

[12] D.C. Montgomery. "Design and analysis of experiments". 2nd Edition. John Wiley and Sons. New York. 1984. 